Check for updates

Cite this: New J. Chem., 2020 44, 18233

Received 3rd August 2020,

Accepted 10th September 2020

DOI: $10.1039 / d 0 n j 03896 b$

rsc.li/njc

\title{
Voltammetric responses at modified electrodes and aggregation effects of two anticancer molecules: irinotecan and sunitinib $\dagger$
}

\author{
Barbara Vercelli, (D) *a Sara Crotti (D) ${ }^{b}$ and Marco Agostini (D) bc
}

\begin{abstract}
The voltammetric responses of two anticancer molecules irinotecan (ITC) and sunitinib (SUN) were investigated at glassy carbon (GC) and indium tin oxide (ITO) electrodes bare or modified with carbon nanotubes (NTs), NT-Nafion composites (NafionNTs) and poly(3,4-ethylenedioxy-thiophene) (PEDOT). Bare $G C$ electrodes revealed to be the most suitable for the detection of drugs at low concentration levels. Differential pulse voltammetry (DPV) analyses showed that the responses of the anticancer molecules are affected by the formation of aggregates. In particular, aggregation of SUN results in the formation of $\mathrm{H}$-aggregates. DPV analysis extended to human plasma samples revealed the drugs in micromolar concentrations with good reproducibility.
\end{abstract}

\section{Introduction}

Irinotecan (ITC, Chart 1) is a semi-synthetic camptothecin derivative and is used as a single agent or in combination with other chemotherapeutics for the treatment of colorectal, ovarian and small cell lung cancers. ${ }^{1}$ Sunitinib (SUN, Chart 1 ) is a novel oral multi-targeted tyrosine kinase inhibitor with antitumor and antiangiogenic activities. ${ }^{2,3}$ The exposure of these drugs to patients is characterized by considerable variation due to patient non-compliance (e.g. due to drug-related toxicity), drug interactions with other co-medication, variability in oral drug availability and many other factors. To solve the inter-individual variability in the pharmacokinetics of ITC and SUN, the evaluation of the drugs levels in plasma during infusion is necessary. Thus, the development of analytical techniques that can monitor the drugs in patients' plasma in real time and which are also rapid, simple and cost effective is a challenging issue for the development of personalized chemotherapy. The analytical approaches usually employed are based on liquid chromatography (LC) followed by ultraviolet visible spectroscopy (UV-vis) ${ }^{4}$ or tandem mass spectrometry

\footnotetext{
${ }^{a}$ Istituto di Chimica della Materia Condensata e di Tecnologie per l'Energia, CNR-ICMATE, Via R. Cozzi 53, 20125 Milano, Italy.

E-mail: barbara.vercelli@cnr.it

${ }^{b}$ Istituto di Ricerca Pediatrica - Città della Speranza, Corso Stati Uniti 4, 35127 Padova, Italy

${ }^{c}$ Sezione di Clinica Chirurgica, Dipartimento di Scienze Chirurgiche, Oncologiche e Gastroenterologiche, Università degli Studi di Padova, Via Giustiniani, 2, 35124 Padova, Italy

$\dagger$ Electronic supplementary information (ESI) available: Cyclic voltammograms. See DOI: 10.1039/d0nj03896b
}

(MS/MS). ${ }^{5-7}$ However, the clinical application of LC is prevented by problems related to cost, portability of the instruments, which also requires highly specialized personnel, and long analysis time. ${ }^{8}$ In this scenario, electrochemical methods could be a challenging alternative because of their simplicity and low cost. ${ }^{9}$ Recent research works have reported on modified electrodes for electrochemical sensors and biosensors for bio-medical applications. ${ }^{10-15}$
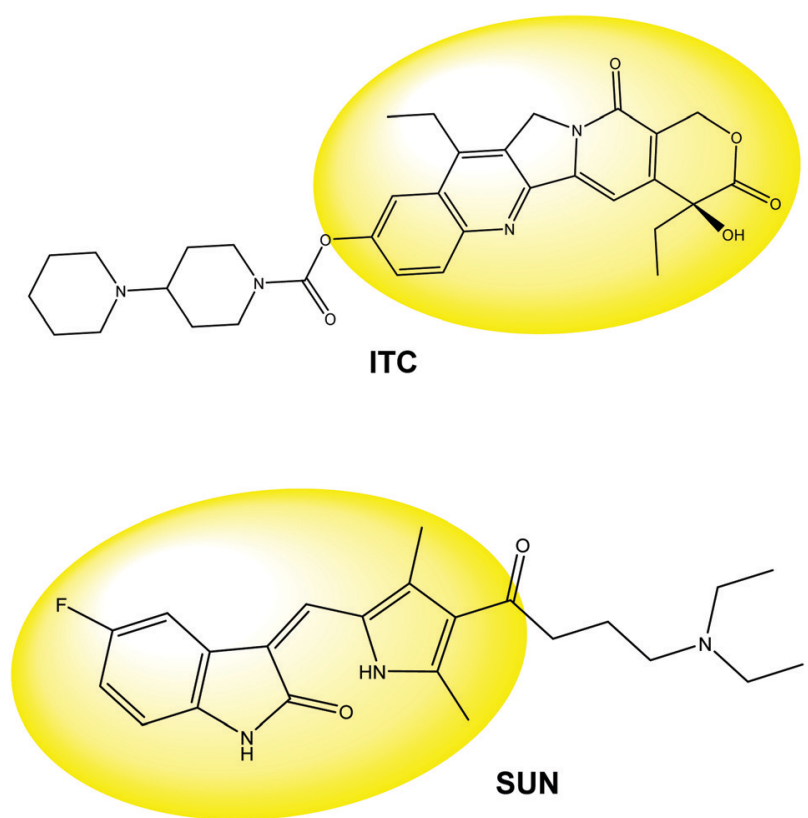

Chart 1 Irinotecan (ITC) and sunitinib (SUN) un-protonated structures. Yellow color highlights the oxidative centers of the molecules. 
In a previous work we reported the electrochemical behavior of ITC and SUN at bare glassy carbon (GC) electrodes. ${ }^{16}$ In the present work we aim to extend our studies to modified electrodes.

A reported work by Madrakian et al. ${ }^{17}$ employs carbon paste electrodes modified with a nanocomposite composed of polyacrilonitrile nanofibers and nickel-zinc ferrite nanoparticles for the electrochemical determination of SUN, here we focused our attention on oxides and carbon-based materials; we did not use metals, such as Pt and $\mathrm{Au}$, because in the potential range of analysis their background signals may lead to wrong interpretation of the anticancer molecule responses. ${ }^{18}$

In particular, we employed indium tin oxide (ITO), carbon nanotubes (NTs), NT-Nafion composites (NafionNTs) and the conducting polymer poly(3,4-ethylenedioxithiophene) (PEDOT). ITO has recently been used as a thin film electrode material in electrochemical biosensors owing to its good electrical conductivity and high transparency to optical wavelengths, rendering it a good candidate for either electrochemical and/ or optical biosensors. ${ }^{19}$ On the other side, NTs have been widely employed in electrode modification because they exhibit good conductivity, high chemical stability, high active surface area and resistance to surface fouling. ${ }^{20}$ Moreover, when they are dispersed in Nafion to form composites, NTs give rise to uniform and stable films which are easy to prepare with good reproducibility and have shown remarkable electrocatalytic properties. ${ }^{21-26}$ Finally, conducting polymers (such as polyanilines, polythiophenes, polypyrroles, etc.) were extensively studied as electrode modifiers because they were found to improve the sensitivity and selectivity of the analytical detection and even lower the detection limit. ${ }^{27}$ In particular, poly(3,4ethylenedioxy-thiophene) (PEDOT) has been the object of great attention owing to its high electrochemical and chemical stability, superior conductivity, good compatibility and adhesion ability with other materials. ${ }^{28,29}$

In this work we employed cyclic voltammetry (CV) to study the electrochemical responses of ITC and SUN at GC and ITO electrodes bare or modified with NTs, NT-Nafion composites and PEDOT. Then we explored the drugs responses at low concentration levels by means of differential pulsed voltammetry (DPV) determinations. In particular, we focused on the influence of the molecular aggregations on their DPV responses. Finally we extended DPV analyses to human plasma samples to detect the anticancer molecules at micromolar levels.

\section{Experimental}

\section{Materials}

Irinotecan (ITC) hydrochloride, sunitinib (SUN) malate, carboxylate-functionalized multi-walled carbon nanotubes (NTs) (avg. diam. $\times$ length: $9.5 \mathrm{~nm} \times 1.5 \mu \mathrm{m}$ ) with a carboxylate content of $>8$ mole percent and Nafion ${ }^{\mathrm{TM}}$ solutions $(5 \%$ solution in lower aliphatic alcohols $+10 \%$ water, 1200 equiv weight) were purchased from Sigma-Aldrich.

ITC and SUN stock solutions $\left(10^{-3} \mathrm{M}\right)$ were prepared by dissolving ITC hydrochloride in deionized water and SUN malate in dimethyl sulfoxide (DMSO) and were stored in a cool and dark place. For SUN the used concentration is limited by the low solubility in water, which is sufficiently high at $\mathrm{pH} 7$ $\left(6 \times 10^{-3} \mathrm{M}\right)$ but decreases sharply with increased $\mathrm{pH}$ down to $1 \times 10^{-4} \mathrm{M}$ at $\mathrm{pH}$ 9. For this reason we have used a $1: 3 \mathrm{v} / \mathrm{v}$ DMSO-water mixture to obtain stable solutions. The strength of all the employed solutions was adjusted to physiological levels by the addition of sodium chloride $0.1 \mathrm{M}$.

Blank human plasma of healthy subjects was obtained from the Tissue Biobank of the first Surgical Clinic of Padua Hospital (Italy). Human plasma solutions were routinely prepared by $1: 10$ dilution of human plasma in $\mathrm{pH} 7$ buffer.

\section{Apparatus and procedure}

Electrochemistry was performed at room temperature in three electrode cells; the counter electrode was platinum and the reference electrode was SCE. The supporting electrolyte was $0.1 \mathrm{M} \mathrm{NaCl}$. Cyclic voltammetry (CV) was performed at a scan rate $0.02 \mathrm{~V} \mathrm{~s}^{-1}$. Differential pulse voltammetry (DPV) response was maximized at pulse amplitude $A=50 \mathrm{mV}$, pulse duration $T=50 \mathrm{~ms}$ and scan rate $v=0.02 \mathrm{~V} \mathrm{~s}^{-1}$. Width of cyclic voltammograms $\Delta E_{\mathrm{p}}$ is calculated as the difference between peak $E_{\mathrm{p}}$ and half-peak $E_{\mathrm{p}} / 2$ potentials, whereas for DPV it is the width at half-height.

Working electrodes were glassy carbon (GC, $0.2 \mathrm{~cm}^{2}$ ) and indium tin oxide (ITO, $2 \mathrm{~cm}^{2}, 20 \Omega \mathrm{sq}^{-1}$, purchased from KINTEC, Hong-Kong) bare or modified as follows:

\section{ITO/NT}

According to the literature,$^{20} 10 \mathrm{mg}$ of NTs were dispersed in $10 \mathrm{ml}$ of dimethylformamide (DMF) under ultrasonic stirring for $2 \mathrm{~h}$ prior to use. By means of a micro-syringe, $10 \mu \mathrm{L}$ of the dispersed NTs were dropped on the electrode surface

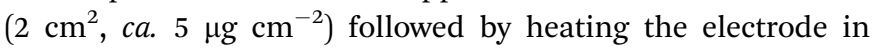
oven at $50{ }^{\circ} \mathrm{C}$ for $15 \mathrm{~min}$.

\section{GC/NafionNT}

According to the literature, ${ }^{22,30} 5 \mathrm{mg}$ of NTs were dispersed in $10 \mathrm{ml}$ of Nafion (wt. $0.1 \%$ ) solution in methanol (MeOH) under ultrasonic stirring for about $30 \mathrm{~min}$. By means of a microsyringe, $10 \mu \mathrm{L}$ of Nafion-dispersed NTs were dropped on the electrode surface $\left(0.2 \mathrm{~cm}^{2}, c a .25 \mu \mathrm{g} \mathrm{cm}{ }^{-2}\right)$ and carefully dried in air.

\section{GC/PEDOT}

According to the literature, ${ }^{31}$ the monomer 3,4-ethylenedioxythiophene (EDOT), 0.1 M in acetonitrile solvent with $0.1 \mathrm{M}$ tetrabutylammonium triflate $\left(\mathrm{Bu}_{4} \mathrm{NCF}_{3} \mathrm{SO}_{3}\right)$, as the supporting electrolyte, was potentiostatically oxidized at $0.9 \mathrm{~V} v s . \mathrm{Ag} / \mathrm{Ag}^{+}$ reference electrode (silver/0.1 $\mathrm{M}$ silver perchlorate in acetonitrile, $0.34 \mathrm{~V}$ vs. SCE). The deposition charge was $50 \mathrm{mC}$ $\mathrm{cm}^{-2}$, with the formation of $c a .100 \mathrm{~nm}$ thick layer on the electrode surface (which corresponds to a polymer amount of $c a .30 \mu \mathrm{g} \mathrm{cm}^{-2}$ for an electrode area of $0.2 \mathrm{~cm}^{2}$ and for a reported PEDOT density value of $\left.1.5 \mathrm{~g} \mathrm{~cm}^{-332,33}\right)$. 
The voltammetric apparatus was Metrohm Autolab 128N potentiostat/galvanostat.

UV-vis spectra were recorded on a PerkinElmer Lambda 35 spectrometer.

\section{Results and discussion}

\section{Cyclic voltammetry}

The analyses were performed in pH 7 and pH 9 buffers, because $\mathrm{pH}$ has a major impact on the stability of both molecules. In particular, it was found that ITC decomposes in $\mathrm{pH}<3$ and $\mathrm{pH}>11$ media. $^{20}$ SUN has an exocyclic alkenyl group and is capable of showing $Z-E$ isomerism (Scheme 1) due to the presence of a double bond between 2-oxindol and the pyrrole ring. ${ }^{34,35}$

The $Z$ isomer is the principal clinical form of the drug and it is stabilized by internal hydrogen bonds between the 2-oxindol and the pyrrole ring. The literature data ${ }^{34}$ show that the $Z$-isomer is not stable in acidic media, because a high concentration of $\mathrm{H}^{+}$ ions in the medium can interfere with the formation of internal hydrogen bonds, thus counteracting the thermodynamic stability of the $Z$-isomer and favoring the prevalence of the $E$-isomer.

\section{ITO}

In pH 9 buffer both molecules exhibited an ill-defined shoulder at $c a .1 \mathrm{~V}$ (Fig. 1 and Fig. S1, ESI $\dagger$ ), with peak current values that are $c a .3$ and 5 times lower, for ITO and SUN, respectively, than the ones at bare GC electrodes (Tables 1 and 2). The width of the cyclic voltammogram $\Delta E_{\mathrm{p}}$ is $c a$. $200 \mathrm{mV}$ for both drugs, a sign of a complex mechanism of electron exchange between ITO and the anticancer molecules. So we modified ITO electrodes with NTs (ITO/NT), in order to optimize the electrochemical response.

\section{ITO/NT}

In $\mathrm{pH} 9$ buffer, ITC exhibited an oxidation peak at $0.67 \mathrm{~V}$, ca. $100 \mathrm{mV}$ less positive than at bare GC electrodes (Fig. 1), with a peak current value that is ca. 2 times lower (Table 1 ). $\Delta E_{\mathrm{p}}$ is $70 \mathrm{mV}$ a sign of an electron transfer process faster than that at

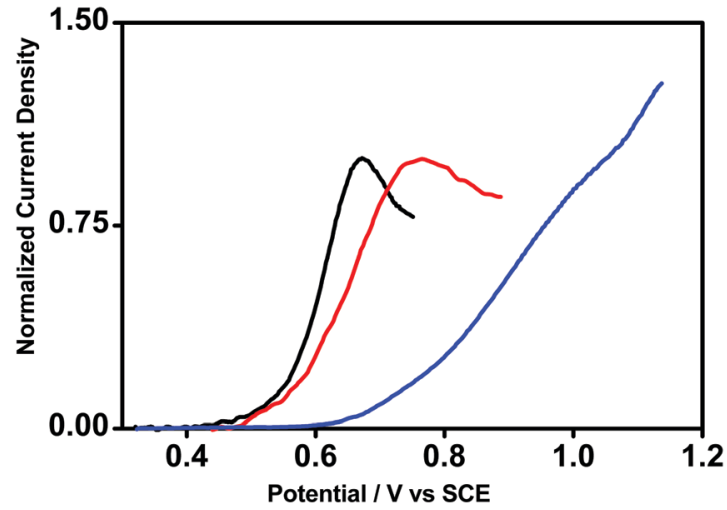

Fig. 1 Normalized single sweep cyclic voltammograms of ITC $10^{-4} \mathrm{M}$ in $\mathrm{pH} 9$ buffer $+\mathrm{NaCl} 0.1 \mathrm{M}$ at: ITO (blue line), ITO/NT (black line) and GC (red line) electrodes. $v=0.02 \mathrm{~V} \mathrm{~s}^{-1}$.

Table 1 Peak current $i_{\mathrm{p}}$, peak potential $E_{\mathrm{p}}$ (vs. SCE) and peak width $\Delta E_{\mathrm{p}}$ for $10^{-4} \mathrm{M}$ ITC in $0.1 \mathrm{M} \mathrm{NaCl} \mathrm{pH} 9$ and 7 buffers at a scan rate of $0.02 \mathrm{~V} \mathrm{~s}^{-1}$

\begin{tabular}{lllll}
\hline Electrode & $\mathrm{pH}$ & $i_{\mathrm{p}} / \mu \mathrm{A} \mathrm{cm}^{-2}$ & $E_{\mathrm{p}} / \mathrm{V}$ & $\Delta E_{\mathrm{p}} / \mathrm{mV}$ \\
\hline GC & 9 & 15 & 0.77 & 120 \\
GC/NafionNT & 9 & 45 & 0.77 & 120 \\
GC/PEDOT & 9 & 70 & 0.70 & 60 \\
ITO/NT & 9 & 8.4 & 0.67 & 70 \\
ITO & 9 & 5 & 1.0 & $>200$ \\
& & & & \\
GC & 7 & 15 & 0.89 & 120 \\
GC/NafionNT & 7 & 45 & 0.89 & 120 \\
GC/PEDOT & 7 & 60 & 0.77 & 60
\end{tabular}

bare GC electrodes. SUN oxidation potentials are $c a .30 \mathrm{mV}$ less positive than at bare GC electrodes (Fig. S1, ESI $\dagger$ ) with comparable peak current values (Table 2). $\Delta E_{\mathrm{p}}$ of the first oxidation process is $60 \mathrm{mV}$, like that at bare GC electrodes. In $\mathrm{pH} 7$ buffer, oxidation peaks of SUN shifted to higher potential by ca. $120 \mathrm{mV}$, but the peak current and $\Delta E_{\mathrm{p}}$ were the same (Table 2). As expected for an oxidation process involving oneproton loss per exchanged electron the peaks shift to higher potential by $60 \mathrm{mV}$ per $\mathrm{pH}$ unit, i.e. passing from $\mathrm{pH} 9$ to $\mathrm{pH} 7$
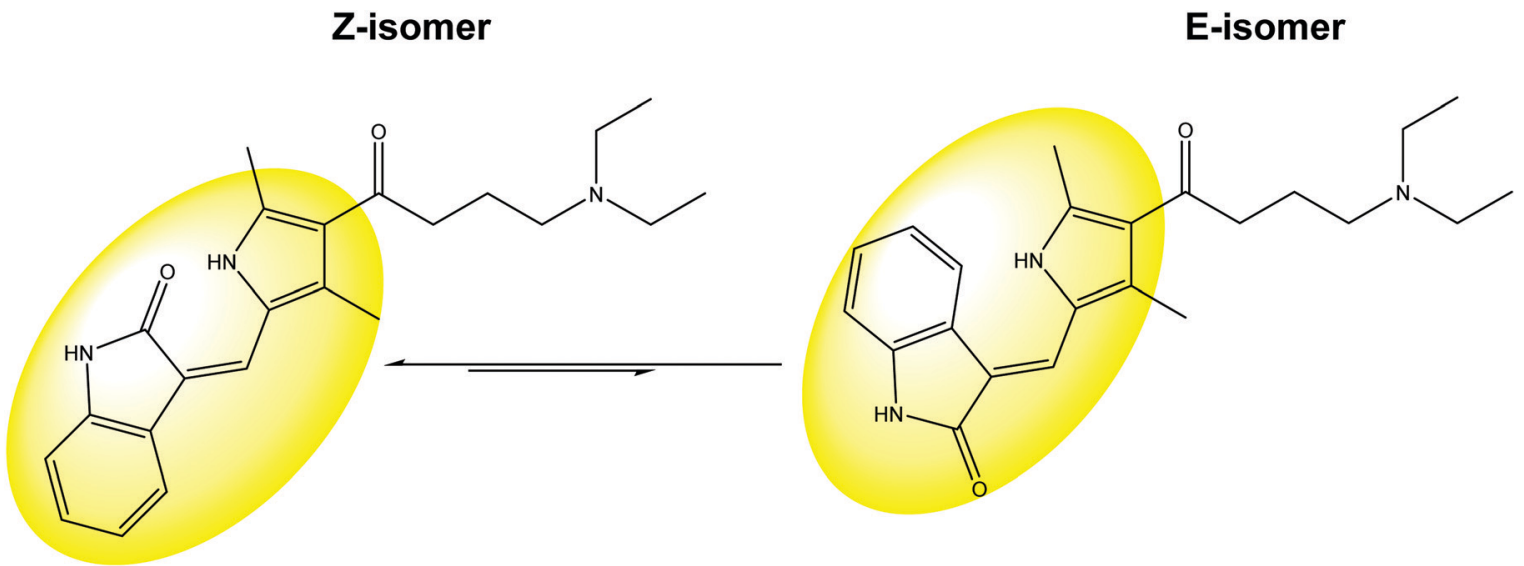

Scheme 1 Structures of SUN Z and E-isomers. Yellow color highlights the oxidative center of the molecule. 
Table 2 Peak current $i_{\mathrm{p}}$, peak potential $E_{\mathrm{p}}$ and peak width $\Delta E_{\mathrm{p}}$ for SUN in $1: 3$ vv DMSO-water $+0.1 \mathrm{M} \mathrm{NaCl} \mathrm{pH} 9$ and $\mathrm{pH} 7$ buffers $\left(10^{-4} \mathrm{M}\right.$ and $4 \times 10^{-5} \mathrm{M}$ SUN concentration, respectively) at a scan rate of $0.02 \mathrm{~V} \mathrm{~s}^{-1}$

\begin{tabular}{lllll}
\hline Electrode & $\mathrm{pH}$ & $i_{\mathrm{p}} / \mu \mathrm{A} \mathrm{cm}^{-2}$ & $E_{\mathrm{p}} / \mathrm{V}$ & $\Delta E_{\mathrm{p}} / \mathrm{mV}$ \\
\hline GC & 9 & 15 & $0.55 ; 0.65$ & 60 \\
GC/NafionNT & 9 & 55 & $0.52 ; 0.64$ & 70 \\
ITO/NT & 9 & 15 & $0.53 ; 0.63$ & 60 \\
ITO & 9 & 3 & 0.80 & 150 \\
& & & & \\
GC & 7 & 15 & $0.67 ; 0.78$ & 70 \\
GC/NafionNT & 7 & 55 & $0.65 ; 0.75$ & 70 \\
ITO/NT & 7 & 15 & $0.65 ; 0.75$ & 70 \\
ITO & 7 & 5 & 0.90 & $c a .200$
\end{tabular}

(see ref. 16 and references therein). However, the NTs deposited on the ITO surface were found to be nonuniform (they formed eye-visible aggregates) and not robust enough to tolerate a standard sticky-tape test (they could be scratched by a simple fingers' touch). So we dispersed NTs in Nafion solutions (GC/ NafionNT), in order to obtain more uniform and mechanically stable films. ${ }^{2,30}$

\section{GC/NafionNT}

In pH 7 buffer, ITC exhibited an oxidation peak at $0.89 \mathrm{~V}$ with peak current values that are $c a .3$ times higher than those at bare GC electrodes (Fig. S2, ESI, $\dagger$ and Table 1). $\Delta E_{\mathrm{p}}$ is $120 \mathrm{mV}$ similar to bare GC electrodes. As observed above for SUN, in $\mathrm{pH}$ 9 buffer the oxidation peak of ITC shifted cathodically by $120 \mathrm{mV}$ by the 2-unit increase of $\mathrm{pH}^{16}$ In both $\mathrm{pH}$ media, SUN exhibited two oxidation peaks separated by ca. $100 \mathrm{mV}$ (Fig. 2a) with peak current values that, similar to ITC, are ca. 3 times higher than those at bare GC electrodes (Table 2). $\Delta E_{\mathrm{p}}$ is $70 \mathrm{mV}$ similar to bare GC electrodes (Table 2). The oxidation peaks shift from $E_{\mathrm{p}}=0.65 \mathrm{~V}$ and $0.75 \mathrm{~V}$ at $\mathrm{pH} 7$ to $0.53 \mathrm{~V}$ and $0.63 \mathrm{~V}$ at $\mathrm{pH} 9$ as observed for ITC.

The effect of $\mathrm{pH}$ on the first peak potential of SUN was investigated in the $\mathrm{pH}$ range of 5-10. SUN responses (Fig S3, $\mathrm{ESI} \dagger)$ showed that, similar to ITC, ${ }^{16,20}$ the peak potential shifts to less positive values with increasing $\mathrm{pH}$. The peak potential also shows a linear dependence on $\mathrm{pH}$ with a negative slope of -50.2 (Fig. S3, inset, ESI $\dagger$ ):

$$
E_{\mathrm{p}}=1018-50.2 \mathrm{pH}(r=0.98107)
$$

In pH 5 buffer SUN shows one oxidation peak at $0.76 \mathrm{~V}$ (Fig. S3, ESI $\dagger$ ), and not two peaks separated by $c a .100 \mathrm{mV}$ as in the $\mathrm{pH}$ range of $7-10$.

A possible explanation may be found in the change in the structure of its oxidation centre (yellow highlighted in Scheme 1) caused by the $Z-E$ isomerism of the molecule. In fact, in acidic media the high concentration of $\mathrm{H}^{+}$ions facilitates the rotation across the exocyclic group in the SUN structure and favours the switch from the $Z$-isomer, the principle clinical form, to the $E$-isomer (Scheme 1). ${ }^{34}$

The active surface area of NTs in GC/NafionNT electrodes was estimated to be around $3 \mathrm{~m}^{2} \mathrm{~g}^{-1}$ by $\mathrm{CV}$, using $\mathrm{K}_{4} \mathrm{Fe}(\mathrm{CN})_{6}$ $10^{-3} \mathrm{M}(0.1 \mathrm{M} \mathrm{NaCl})$. The estimated value is two orders of magnitude lower than the one reported for commercial NTs ( ca. $400 \mathrm{~m}^{2} \mathrm{~g}^{-136}$ ) and clearly shows that the active area of NTs is limited by the dispersion in Nafion.

\section{GC/PEDOT}

In pH 7 buffer, ITC exhibited a peak at $0.77 \mathrm{~V}$ (Fig. 2b) with a peak current value that is $c a .4$ times higher than that at bare GC electrodes (Table 1). $\Delta E_{\mathrm{p}}$ is $60 \mathrm{mV}$ a sign of an electron transfer process faster than the one at bare GC electrodes.

In $\mathrm{pH} 9$ buffer, the oxidation peak of ITC shifted to $0.70 \mathrm{~V}$, but the peak current and $\Delta E_{\mathrm{p}}$ were the same (Table 1$)$. Unfortunately, CV responses of SUN are ill-defined in both $\mathrm{pH}$-media. We suppose that DMSO, added to obtain stable SUN solutions, ${ }^{16}$ may cause the swelling of the PEDOT film, thus leading to a difficult interpretation of the responses of the molecule.

The above reported results show that both drugs exhibited promising $\mathrm{CV}$ responses at ITO/NT and at GC/NafionNT electrodes. In fact, at ITO/NT electrodes the peak potential values of ITC and SUN are100 $\mathrm{mV}$ and $30 \mathrm{mV}$, respectively, less positive

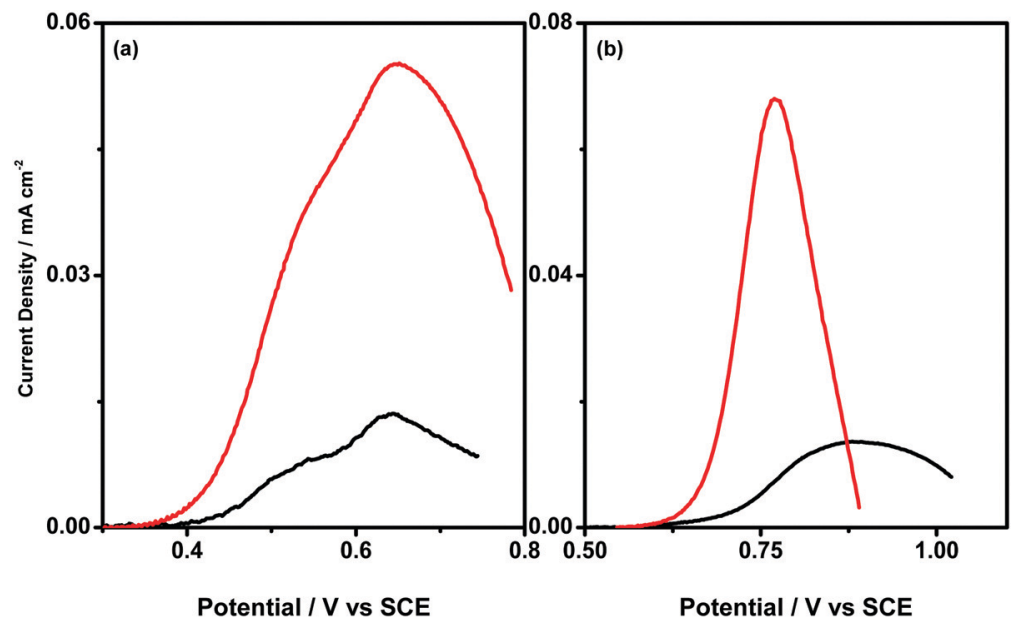

Fig. 2 Single sweep cyclic voltammograms of: (a) SUN $4 \times 10^{-5} \mathrm{M}$ in $1: 3 \mathrm{vv} \mathrm{DMSO/water} \mathrm{pH} 9$ buffer $+0.1 \mathrm{NaCl}$ at GC (black line) and CG/NafionNT (red line) electrodes, and (b) ITC $10^{-4} \mathrm{M}$ in pH 9 buffer $+\mathrm{NaCl} 0.1 \mathrm{M}$ at GC (black line) and GC/PEDOT (red line) electrodes. $v=0.02 \mathrm{~V} \mathrm{~s}$. 
than those at bare GC electrodes and the electron transfer rate of ITC is faster (Tables 1 and 2). However, the NTs deposited on ITO appeared neither uniform nor sufficiently robust. So we dispersed the NTs in Nafion solutions to obtain more uniform and mechanically stable films. ${ }^{22,30}$ In fact, at GC/NafionNT electrodes the peak current values of both drugs are three times higher than the ones at bare GC electrodes (Tables 1 and 2), but the estimated active surface area of NTs is limited by the dispersion in Nafion. Moreover, the signal-to-noise ratio at GC/NafionNT electrodes, estimated from the faradaic and the capacitive currents, is higher than the one at bare GC electrodes. Thus, from these considerations, we selected the bare GC electrodes for the determination of the drugs in low concentration levels, as described in the following.

\section{Differential pulse voltammetry}

For ITC, the DPV response (Fig. 3a) exhibits a single oxidation peak. The peak potential shifts to less positive values as the concentration is decreased, moving from $0.667 \mathrm{~V}$ to 0.621 and $0.615 \mathrm{~V}$ from $10^{-4} \mathrm{M}$ to $10^{-5}$ and $10^{-6} \mathrm{M}$ (at pH 9, see Table 3).

The peak width is $140 \mathrm{mV}$ at $10^{-4} \mathrm{M}$ concentration but decreases to $c a .80 \mathrm{mV}$ in diluted solutions. The theoretical width of the DPV response is $90 \mathrm{mV}$ for a fast electron transfer and $c a .150 \mathrm{mV}$, for a slow transfer. ${ }^{37}$ Thus it appears that the transfer rate increases in the diluted solutions. The peak current is essentially linear in the $10^{-6} \mathrm{M}$ to $10^{-4} \mathrm{M}$ concentration range (Fig. $\mathrm{S} 4, \mathrm{ESI} \dagger$ ) and the corresponding regression equation is $\mathrm{y}=2.4 \times 10^{5}+0.8, R^{2}=0.993$. The oxidation peak in diluted solutions shifts from $E_{\mathrm{p}}=0.71 \mathrm{~V}$ at $\mathrm{pH} 7$ to $E_{\mathrm{p}}=0.61 \mathrm{~V}$ at pH 9 as observed in cyclic voltammetry.

The DPV response of SUN in 1:3 vv DMSO/water (Fig. 3b) exhibits a single oxidation peak at $E_{\mathrm{p}}=0.65 \mathrm{~V}$ at $\mathrm{pH} 7$ in $10^{-4} \mathrm{M}$ solution. As summarized in Table 3, in more diluted solutions $\left(10^{-5} \mathrm{M}\right.$ and $\left.10^{-6} \mathrm{M}\right)$ the response is split into a twin peak signal at $E_{\mathrm{p}}=0.60 \mathrm{~V}$ and $0.70 \mathrm{~V}$ (at pH 7); at $\mathrm{pH} 9$ the twin peaks are shown at $E_{\mathrm{p}}=0.49 \mathrm{~V}$ and $E_{\mathrm{p}}=0.59 \mathrm{~V}$, i.e. with the cathodic shift observed in cyclic voltammetry. The peak width
Table 3 Peak current $i_{p}$, peak potential $E_{\mathrm{p}}$ and peak width $\Delta E_{\mathrm{p}}$ for a typical DPV analysis of ITC and SUN in $0.1 \mathrm{M} \mathrm{NaCl}$ on the GC electrode at various concentrations, $C$

\begin{tabular}{lllll}
\hline$C / \mathrm{M}$ & $\mathrm{pH}$ & $i_{\mathrm{p}} / \mu \mathrm{A} \mathrm{cm}^{-2}$ & $E_{\mathrm{p}} / \mathrm{V}$ & $\Delta E_{\mathrm{p}} / \mathrm{mV}$ \\
\hline ITC & & & & \\
$10^{-4}$ & 9 & 25 & 0.667 & 140 \\
$10^{-5}$ & 9 & 4 & 0.621 & 80 \\
$10^{-6}$ & 9 & 0.3 & 0.615 & 80 \\
SUN & & & & \\
$10^{-4}$ & 7 & 30 & 0.646 & 80 \\
$10^{-5}$ & 7 & $4 ; 6$ & $0.595 ; 0.682$ & 80 \\
$10^{-6}$ & 7 & $0.3 ; 0.6$ & $0.585 ; 0.685$ & 80 \\
\end{tabular}

is ca. $80 \mathrm{mV}$ at all concentrations indicating fast electron transfer in all cases. Also in the case of SUN the peak current is linear in the $10^{-6} \mathrm{M}$ to $10^{-4} \mathrm{M}$ concentration range (Fig. S4, ESI $\dagger$ ), with a detection limit of $c a \cdot 10^{-7} \mathrm{M}$, the corresponding regression equation is $y=2.9 \times 10^{5}+0.5, R^{2}=0.997$.

DPV analysis of SUN was also performed in $10^{-5} \mathrm{M}$ and $10^{-6} \mathrm{M}$ solutions at pH 7 obtained by dilution of the standard $10^{-4} \mathrm{M}$ SUN solution into DMSO-free buffer. Lowering the DMSO content from $25 \%$ to $2.5 \%$ makes the single peak become a twin response peak (Fig. 4a). A possible explanation may be found in the formation of molecular aggregates as described in the following.

\section{Aggregation effects}

In contrast to organic solvents, aggregates (mainly dimers) are formed by ITC in water. ${ }^{38}$ It is therefore suggested that aggregation causes the observed potential shifts and width changes in the ITC DPV signals with concentration, as when the oxidation charge-transfer rate is strongly decreased in the aggregated forms. The splitting of the DPV response of SUN with the decrease of concentration could be in principle attributed to analogous aggregation effects. The fact that the splitting is removed by increasing the DMSO content is in line with this suggestion (Fig. 4a). Also, the reported data on the aggregation of conjugated oligomers show the significant splitting of their oxidation responses upon aggregation. ${ }^{39}$ Aggregation of ITC
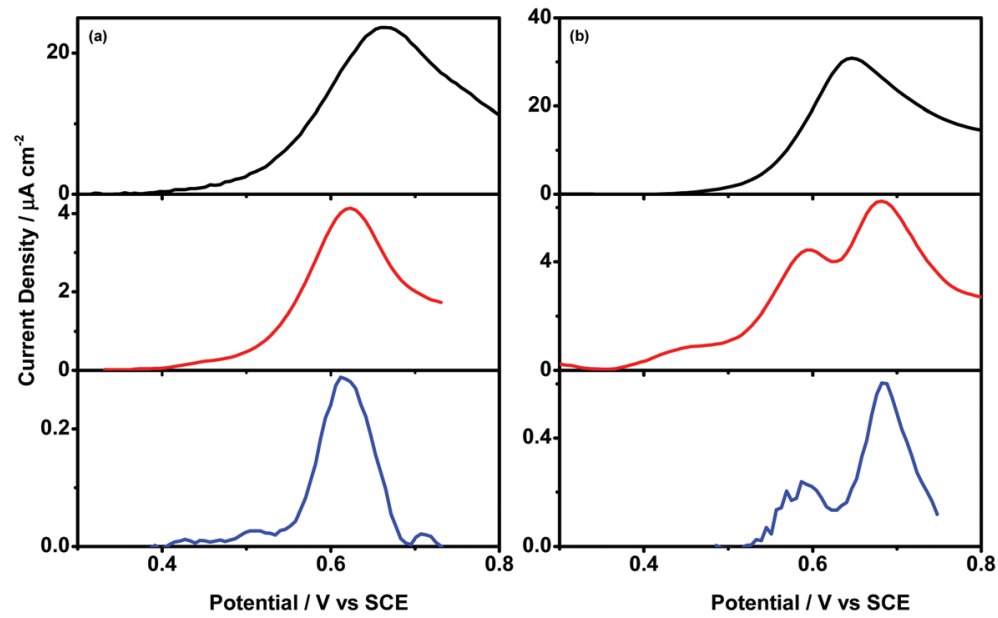

Fig. 3 Differential pulse voltammetry of: (a) ITC $10^{-4} \mathrm{M}$ (black line), $10^{-5} \mathrm{M}$ (red line) and $10^{-6} \mathrm{M}$ (blue line) in pH 9 buffer $+0.1 \mathrm{NaCl}$; and (b) SUN $10^{-4} \mathrm{M}$ (black line), $10^{-5} \mathrm{M}$ (red line) and $10^{-6} \mathrm{M}$ (blue line) in $\mathrm{pH} 7$ buffer $+0.1 \mathrm{NaCl}$ on the $\mathrm{GC}$ electrode. 

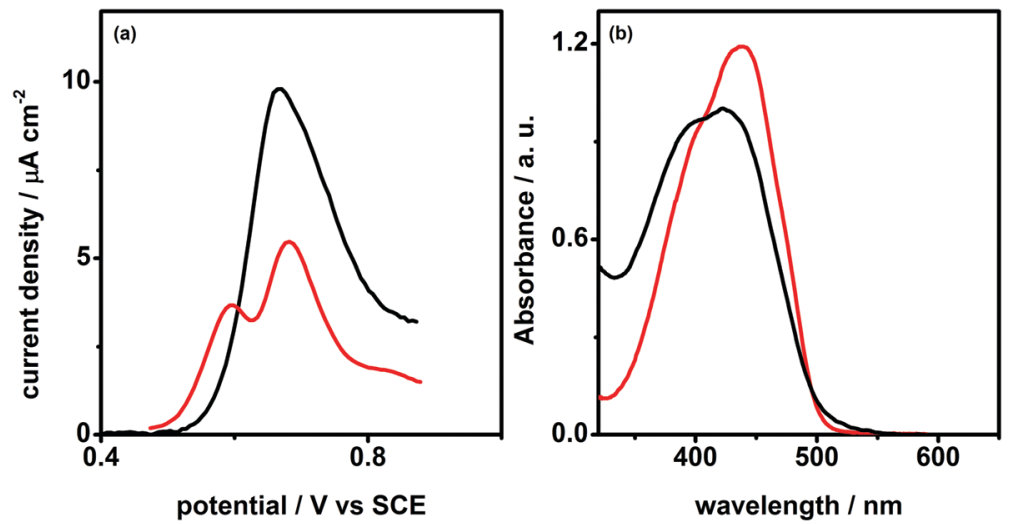

Fig. 4 (a) Differential pulse voltammetry of SUN $10^{-5} \mathrm{M}$ in pH 7 buffer $+0.1 \mathrm{NaCl}$ with 25\% DMSO (black line) and $2.5 \%$ DMSO (red line) at the CG electrode. (b) UV-vis spectra of SUN $10^{-5} \mathrm{M}$ in water (black line) and in 1:3 v/v DMSO/water mixture (red line).

was previously revealed by the UV-vis spectra of aqueous solution which exhibited an appreciable hypochromic effect when the concentration of the solute is increased from $10^{-5} \mathrm{M}$ to $10^{-3} \mathrm{M}$, involving the two main absorption maxima at 355 and $368 \mathrm{~nm}$. The absorption at the low-energy peak exhibited a conspicuous decrease of intensity when the concentration of the solute is increased, whereas that at the high energy side did not change. ${ }^{38}$ We evidenced aggregation, also, for SUN by UV-vis spectroscopy. The spectrum of saturated water solution $\left(c a .10^{-5} \mathrm{M}\right.$ ) was compared with the spectrum obtained in $1: 3 \mathrm{wv}$ DMSO/water solution (Fig. $4 \mathrm{~b}$ ). The highest energy band measured ( $c a .400 \mathrm{~nm}$ ) in water becomes a shoulder in $\mathrm{DMSO} /$ water solution where the organic solvent disaggregates the dimers. The picture resembles that of ITC with dilution. ${ }^{38}$ In the case of SUN, the maximum peak is shifted hypsochromically from 440 to $423 \mathrm{~nm}$ suggesting that aggregation of the planar conjugated molecules results in the production of $\mathrm{H}$-aggregate forms. ${ }^{40}$

\section{Human plasma}

Blood plasma samples were obtained from healthy volunteers who had not received any treatment, including operation,
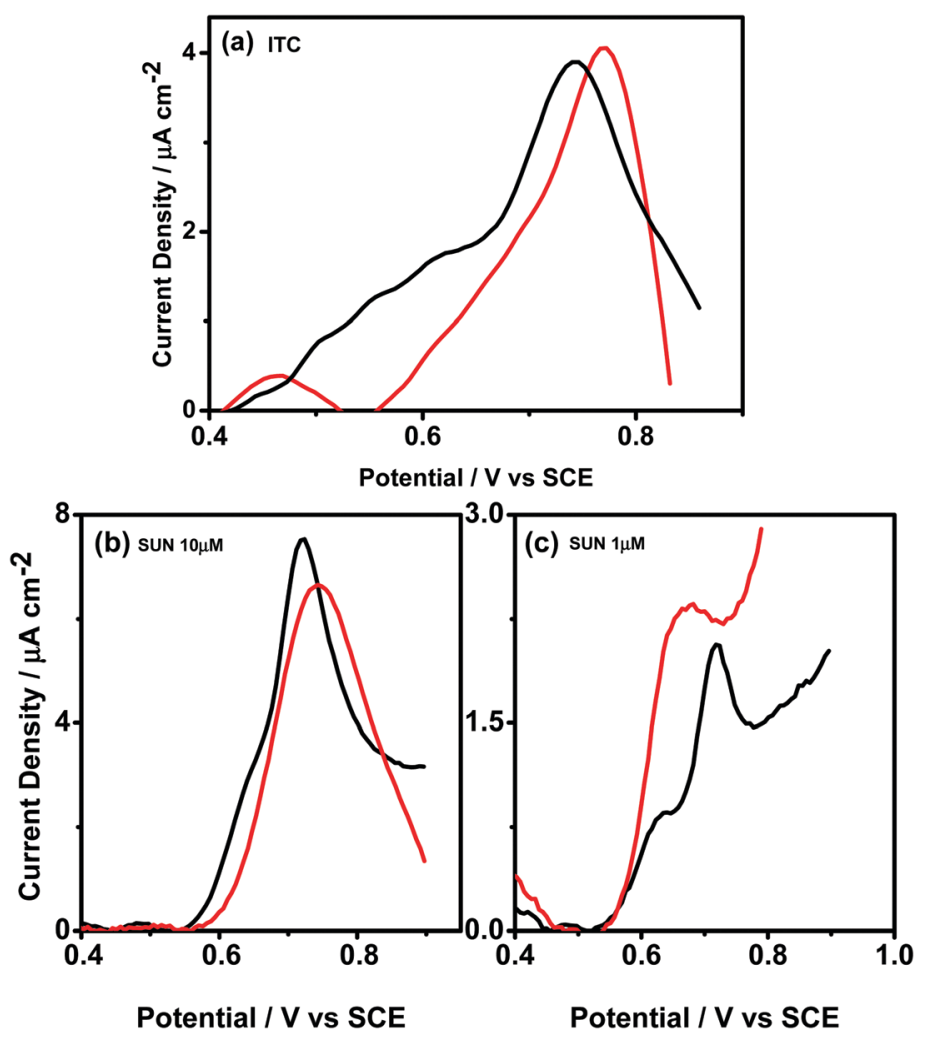

Fig. 5 Differential pulse voltammetry at GC electrodes of: (a) ITC, and (b) and (c) SUN in pH 7 buffer $+0.1 \mathrm{M} \mathrm{NaCl}$ before (black line, pure solutions) and after (red line, plasma samples) human plasma addition. 
chemotherapy or radiation therapy. Plasma samples were routinely prepared by adding human plasma ( $1: 10$ dilution) to pH 7 buffers and then spiked with known amounts of anticancer drugs ( $5 \mu \mathrm{M}$ for ITC, $10 \mu \mathrm{M}$ and $5 \mu \mathrm{M}$ for SUN). DPV response of ITC in human plasma samples exhibits a single oxidation peak at $0.77 \mathrm{~V}$, i.e. $30 \mathrm{mV}$ more positive than that in pure solutions (Fig. 5a). In the case of SUN, the DPV twin peak signal observed in pure solutions (Fig. 5b and c) becomes a single oxidation response in human plasma samples. The peak current values of both drugs are comparable with those observed in pure solutions, for a given drug amount, showing that there are no significant interferences from plasma excipients. ${ }^{34}$ The relative standard deviations of the peak current in plasma samples are $4 \%$ and $6 \%(n=3)$ for ITC and SUN, respectively, demonstrating the good reproducibility of the measurements.

\section{Conclusions}

We have investigated the responses of the anticancer molecules irinotecan and sunitinib at modified electrodes in $\mathrm{pH} 7$ and pH 9 media by CV determinations. We observed that the bare GC electrodes revealed to be more suitable for the detection of the drugs in low concentration levels. In fact at GC/NafionNT electrodes the peak current values of both drugs are three times higher than the ones at bare GC electrodes, but the estimated active surface area of NTs is limited by the dispersion in Nafion. On the other side, at ITO/NT electrodes the peak potential values of ITC and SUN are, respectively, $100 \mathrm{mV}$ and $30 \mathrm{mV}$ less positive than those at bare GC electrodes and the electron transfer rate of ITC is faster, but, for the employed electrode areas, the deposited NTs appeared neither uniform nor significantly robust. A possible alternative, which will be the object of further studies, is to deposit NT films on smaller-dimension electrodes, i.e. micro-electrodes.

DPV analyses showed that the drug responses are affected by molecular aggregate formation. In particular, the oxidation charge-transfer rate of ITC is strongly decreased in the aggregated forms, while the DPV response of SUN splits into a twin peak signal upon aggregate formation. Moreover, the aggregation of SUN results in the production of $\mathrm{H}$-aggregate forms. DPV measurements were successfully extended to human plasma samples and revealed both drugs in micromolar concentration levels, with good reproducibility. To the best of our knowledge this is the first study on the voltammetric responses of two anticancer molecules at bare glassy carbon electrodes, also extended to human plasma samples.

\section{Compliance with ethical standards}

The study was conducted according to the principles expressed in the Declaration of Helsinki. Blank human plasma of healthy subjects was obtained from the Tissue Biobank of the first Surgical Clinic of Padua Hospital (Ethical committee of Centro Oncologico Regionale/I.O.V., Azienda Ospedaliera di Padova, approved protocol number: P.448).

\section{Conflicts of interest}

There are no conflicts to declare.

\section{Acknowledgements}

With this work we want to remember Dr Gianni Zotti, who suddenly passed away on 15th of April 2020. Dr Zotti was an esteemed electrochemist of CNR, Padova. In 1984, he produced the first conjugated polymer films by the cathodic route, which opened the way to the electrochemistry of conducting polymers. His studies concerned the development of conjugated polymers and molecular materials with specific electronic properties for application in electronics, photonics, energy conversion, and sensors. Apart from his achievements in science, Gianni was an extraordinary human being with an amazing intellect, sense of humor, enthusiasm and a warm, generous personality. We will miss him deeply. We also would like to thank Dr Anna Berlin of CNR, Milan, for the helpful discussions. We are indebted to the Italian Government (MUR) for financial support.

\section{Notes and references}

1 E. Calandra, S. Crotti, D. Nitti, M. Roverso, G. Toffoli, E. Marangon, B. Posocco, P. Traldi and M. Agostini, The development of a matrix-assisted laser desorption/ionization (MALDI)-based analytical method for determination of irinotecan levels in human plasma: preliminary results, J. Mass Spectrom., 2015, 50, 959-962.

2 S. Faivre, C. Delbaldo, K. Vera, C. Robert, S. Lozahic, N. Lassau, C. Bello, S. Deprimo, N. Brega, G. Massimini, J.-P. Armand, P. Scigalla and E. Raymond, Safety, pharmacokinetic, and antitumor activity of SU11248, a novel oral multitarget tyrosine kinase inhibitor, in patients with cancer, J. Clin. Oncol., 2006, 24(1), 25-35.

3 N. A. G. Lankheet, J. S. L. Kloth, C. G. M. Gadellaa-van Hooijdonk, G. A. Cirkel, R. H. J. Mathijssen, M. P. J. K. Lolkema, J. H. M. Schellens, E. E. Voest, S. Sleijfer, M. J. A. de Jonge, J. B. A. G. Haanen, J. H. Beijnen, A. D. R. Huitema and N. Steeghs, Pharmacokinetically guided sunitinib dosing: a feasibility study in patients with advanced solid tumours, B. J. Cancer., 2014, 110(10), 2441-2449.

4 T. Bansala, A. Awasthi, M. Jaggi, R. K. Khar and S. Talegaonkar, Development and validation of reversed phase liquid chromatographic method utilizing ultraviolet detection for quantification of irinotecan (CPT-11) and its active metabolite, $\mathrm{SN}-38$, in rat plasma and bile samples: Application to pharmacokinetic studies, Talanta, 2008, 76, 1015-1021.

5 X. Chen, C. J. Peer, R. Alfaro, T. Tian, S. D. Spencer and W. D. Figg, Quantification of irinotecan, SN38, and SN38G in human and porcine plasma by ultra high-performance liquid chromatography-tandem mass spectrometry and its application to hepatic chemoembolization, J. Pharm. Biomed. Anal., 2012, 62, 140-148. 
6 G. Ahn, D. M. Park, J. W. Park, H.-Y. Kim, J.-Y. Cho, S.-J. Rhee, I.-J. Jang and H. Kyun Kim, A rapid, simple and reliable HPLC-triple quadrupole tandem mass spectrometer method for a simultaneous quantification of irinotecan and its active metabolite 7-ethyl-10-hydroxycamptothecin (SN38) in mouse plasma, Biomed. Chromatogr., 2014, 28, 919-922.

7 E. Marangon, M. Buzzo, B. Posocco, S. Gagno, M. Zanchetta, V. Iacuzzi, A. S. Poetto, M. Guardascione, L. Giodini and G. Toffoli, A new high-performance liquid chromatographytandem massspectrometry method for the determination of sunitinib andN-desethyl sunitinib in human plasma: Light-induced isomerismovertaking towards therapeutic drug monitoring in clinical routine, J. Pharm. Biomed. Anal., 2020, 179, 112949.

8 A. Paci, G. Veal, C. Bardin, D. Levêque, N. Widmer, J. J. Beijnen, A. Astieri and E. Chatelut, Review of therapeutic drug monitoring of anticancer drugs part 1 - cytotoxics, Eur. J. Cancer, 2014, 50, 2010-2019.

9 B. Pejcic, R. De Marco and G. Parkinson, The role of biosensors in the detection of emerging infectious diseases, Analist, 2006, 131, 1079.

10 N. P. Shetti, S. D. Bukkitgar, K. R. Reddy, Ch. V. Ch. Venkata Reddy and T. M. Aminabhav, ZnO-based nanostructured electrodes for electrochemical sensors and biosensors in biomedical applications, Biosens. Bioelectron., 2019, 141, 111417.

11 N. P. Shetti, A. Mishra, S. Basu, R. J. Mascarenhas, K. R. Reddy and T. M. Aminabhavi, Skin-Patchable Electrodes for Biosensor Applications: A Review, ACS Biomater. Sci. Eng., 2020, 6, 1823-1835.

12 N. P. Shetti, S. J. Malode, D. S. Nayak, K. R. Kakarla Raghava Reddy, Ch. V. Reddy and K. Ravindranadh, Silica gelmodified electrode as an electrochemical sensor for the detection of acetaminophen, Microchem. J., 2019, 150, 104206.

13 G. Manasa, J. Ronald, R. J. Mascarenhasa and B. M. Basavaraj, Sensitively-selective determination of Propyl Paraben preservative based on synergistic effects of polyaniline-zinc-oxide nano-composite incorporated into graphite paste electrode, Colloids Surf., B, 2019, 184, 110529.

14 N. P. Shetti, M. M. Shanbhag, S. J. Malodea, R. K. Srivastava and K. R. Reddy, Amberlite XAD-4 modified electrodes for highly sensitive electrochemical determination of nimesulide in human urine, Microchem. J., 2020, 153, 104389.

15 M. G. Madhusudhana, A. K. Bhakta, Z. Mekhalif and R. J. Mascarenhas, Bismuth-nanoparticles decorated multi-wallcarbon-nanotubes cast-coated on carbon paste electrode; an electrochemical sensor for sensitive determination of Gallic Acid at neutral pH, Mater. Sci. Energy Technol., 2020, 3, 174-182.

16 B. Vercelli, G. Zotti and A. Berlin, Electrochemistry of conjugated planar anticancer molecules: Irinotecan and Sunitinib, Electrochim. Acta, 2017, 231, 336-343.

17 A. Yarahmadi, T. Madrakian, A. Afkhami and N. R. Jalal, Electrochemical Determination of Sunitinib in Biological Samples Using Polyacrylonitrile Nanofibers/Nickel-Zinc-Ferrite Nanocomposite/Carbon Paste Electrode, J. Electrochem. Soc., 2019, 166(14), B1268-B1275.
18 B.-C. Lai, J.-G. Wu and S.-C. Luo, Revisiting Background Signals and the Electrochemical Windows of Au, Pt, and GC Electrodes in Biological Buffers, ACS Appl. Energy Mater., 2019, 2, 6808-6816.

19 M.-S. Wu, D.-J. Yuan, J.-J. Xu and H.-Y. Chen, Sensitive Electrochemiluminescence Biosensor Based on Au-ITO Hybrid Bipolar Electrode Amplification System for Cell Surface Protein Detection, Anal. Chem., 2013, 85, 11960-11965.

20 N. Karadas, S. Sanli, B. Akmese, B. Dogan-Topal, A. Can and S. A. Ozkan, Analytical application of polymethylene bluemultiwalled carbon nanotubes modified glassy carbon electrode on anticancer drug irinotecan and determination of its ionization constant value, Talanta, 2013, 115, 911-919.

21 L. V. Tarditto, F. J. Arévalo, M. A. Zon, H. G. Ovando, N. R. Vettorazzi and H. Fernández, Electrochemical sensor for the determination of enterotoxigenic Escherichia coli in swine feces using glassy carbon electrodes modified with multiwalled carbon nanotubes, Microchem. J., 2016, 127, 220-225.

$22 \mathrm{~K}$. Wu and S. Hu, Electrochemical Study and Selective Determination of Dopamine at a Multi-Wall Carbon Nanotube-Nafion Film Coated Glassy Carbon Electrode, Microchim. Acta, 2004, 144, 131-137.

23 R. X. Guo, Q. Xu, D. Y. Wang and X. Y. Hu, Trace determination of clenbuterol with an MWCNT-Nafion nanocomposite modified electrode, Microchim. Acta, 2008, 161, 265-272.

24 D. Sun and Z. Sun, Electrochemical determination of $\mathrm{Pb}^{2+}$ using a carbon nanotube/Nafion composite film-modified electrode, J. Appl. Electrochem., 2008, 38, 1223-1227.

25 Y. C. Tsai, J. M. Chen, S. C. Li and F. Marken, Electroanalytical thin film electrodes based on a Nafion ${ }^{\mathrm{TM}}$ - multiwalled carbon nanotube composite, Electrochem. Commun., 2004, 6, 917-922.

26 W. Huang, C. Yang and S. Zhang, Simultaneous determination of 2-nitrophenol and 4-nitrophenol based on the multiwall carbon nanotubes Nafion-modified electrode, Anal. Bioanal. Chem., 2003, 375, 703-707.

27 A. Bello, M. Giannetto, G. Mori, R. Seeber, F. Terzi and C. Zanardi, Optimization of the DPV potential waveform for determination of ascorbic acid on PEDOT-modified electrodes, Sens. Actuators, B, 2007, 121, 430-435.

28 S. Senthil Kumar, J. Mathiyarasu, K. L. N. Phani and V. Yegnaraman, Simultaneous determination of dopamine and ascorbic acid on poly(3,4-ethylenedioxythiophene) modified glassy carbon electrode, J. Solid State Electrochem., 2006, 10, 905-913.

29 F. Sekli-Belaidi, P. Temple-Boyer and P. Gros, Voltammetric microsensor using PEDOT-modified gold electrode for the simultaneous assay of ascorbic and uric acids, J. Electroanal. Chem., 2010, 647, 159-168.

30 J. Wang, M. Musameh and Y. Lin, Solubilization of Carbon Nanotubes by Nafion toward the Preparation of Amperometric Biosensors, J. Am. Chem. Soc., 2003, 125, 2408-2409.

31 G. Zotti, S. Zecchin, G. Schiavon and L. B. Groenedaal, Conductive and Magnetic Properties of 3,4-Dimethoxyand 3,4-Ethylenedioxy-Capped Polypyrrole and Polythiophene, Chem. Mater., 2000, 12, 2996-3005. 
32 A. Baba, J. Lbben, K. Tamada and W. Knoll, Optical Properties of Ultrathin Poly(3,4-ethylenedioxythiophene) Films at Several Doping Levels Studied by In Situ Electrochemical Surface Plasmon Resonance Spectroscopy, Langmuir, 2003, 19, 9058-9064.

33 L. Niua, C. Kvarnströma, K. Fröbergb and A. Ivaska, Electrochemically controlled surface morphology and crystallinity in poly(3,4-ethylenedioxythiophene) films, Synth. Met., 2001, 122, 425-429.

34 M. Padervand, S. Ghaffari, H. Attar and M. M. Nejad, Reverse phase HPLC determination of Sunitinib Malate using UV detector, its isomerisation study, method development and validation, J. Anal. Chem., 2017, 72(5), 567-574.

35 B. Posocco, M. Buzzo, L. Giodini, S. Crotti, S. D’Aroncoc, P. Traldi, M. Agostini, E. Marangon and G. Toffoli, Analytical aspects of sunitinib and its geometric isomerism towards therapeutic drug monitoring in clinical routine, J. Pharm. Biomed. Anal., 2018, 160, 360-367.
36 A. Peigney, C. Laurent, E. Flahaut, R. R. Bacsa and A. Rousset, Specific surface area of carbon nanotubes and bundles of carbon nanotubes, Carbon, 2001, 39, 507-514 and reference therein.

37 T. R. Brumleve and J. Osteryoung, Theory of Differential Normal Pulse Voltammetry in the Alternating Pulse Mode for Totally Irreversible Electrode Reactions, Anal. Chem., 1981, 53, 988-991.

38 R. Aiyama, Y. Nagai, S. Sawada, T. Yokokura, H. Itokawa and M. Nakanishi, Determination of Self-Association of Irinotecan Hydrochloride (CPT-11) in Aqueous Solution, Chem. Pharm. Bull., 1992, 40, 2810-2813.

39 K. M. Knoblock, C. J. Silvestri and D. M. Collard, Stacked Conjugated Oligomers as Molecular Models to Examine Interchain Interactions in Conjugated Materials, J. Am. Chem. Soc., 2006, 128, 13680-13681.

40 A. Eisfeld and J. S. Briggs, The J- and H-bands of organic dye aggregates, Chem. Phys., 2006, 376-384. 